\title{
Utilidad de biopsias endoscópicas en pacientes con diarrea crónica no inflamatoria y colonoscopia normal
}

\author{
Luis Alberto López-Valenzuela ${ }^{*}$, Enrique Murcio-Pérez², Óscar Víctor Hernández-Mondragón², \\ Gerardo Blanco-Velasco ${ }^{2}$, David Espinosa-Saavedra', Raquel Palos-Cuellar ${ }^{1}$ y María Barragán-Valarezo ${ }^{1}$ \\ ${ }^{1}$ Médico en adiestramiento endoscópico; ${ }^{2}$ Médico adscrito al Servicio de Endoscopia. UMAE Hospital de Especialidades UMAE Siglo XXI, Ciudad \\ de México, México
}

\section{Resumen}

Introducción: La diarrea crónica se define como un aumento en la frecuencia y volumen de heces con disminución en la consistencia y duración mayor de 4 semanas. La colonoscopia forma parte del arsenal diagnóstico en pacientes con diarrea crónica no inflamatoria (DCNI), sin embargo en estos pacientes la mucosa de colon puede ser normal aun albergando una patología. El rendimiento diagnóstico de la colonoscopia es variable 7-31\%, pero puede proporcionar un diagnóstico definitivo y un tratamiento específico. Objetivo: Evaluar la utilidad de las biopsias endoscópicas en pacientes con diarrea crónica no inflamatoria y colonoscopia normal. Material y métodos: Se realizó un estudio retrolectivo, transversal, descriptivo y observacional en el periodo de Enero 2013 a Enero 2018. Criterios de inclusión: hombres y mujeres entre 18-90 años con $D C N I>4$ semanas, con análisis de laboratorio iniciales normales (hemoglobina, proteína $C$ reactiva, hormona estimulante de tiroides, albúmina), y colonoscopia normal. Criterios de exclusión: pacientes con menos de 4 biopsias de cada segmento derecho e izquierdo de colon, hemorragia de tubo digestivo evidente, examen coprológico con leucocitos fecales y/o sangre oculta en heces positiva, coproparasitoscópicos positivos, coprocultivos positivos, anemia por déficit de hierro, virus de inmunodeficiencia humana/sida; inmunosupresión por fármacos, quimioterapia, neoplasia digestiva, enfermedad inflamatoria intestinal, consumo crónico de AINE por > 6 semanas, cirugía previa de colon, colonoscopia incompleta. Análisis estadístico: Las variables cualitativas se presentan en porcentajes y frecuencias. Para las asociaciones entre variables cuantitativas se utilizó $U$ de Mann-Whitney o t de Student. Para variables cualitativas Chi cuadrada o prueba exacta de Fisher. Se consideró una $p<0.05$ estadísticamente significativo. Se utilizó SPSS versión 21. Resultados: Se revisaron 349 pacientes, 141/349 cumplieron con criterios de inclusión y fueron analizados, colonoscopia con biopsias tuvo una utilidad para un diagnóstico específico en 25/141 (17.7\%). Estos hallazgos fueron colitis eosinofílica 11/141 (7.8\%), colitis linfocitica 9/141 (6.4\%), colitis ulcerativa crónica idiopática 3/141 (2.1\%), enfermedad de Crohn 1/141 (0.7\%), melanosis coli 1/141 $(0.7 \%)$. La evolución de la diarrea $<6$ meses tiene mayor probabilidad de un diagnóstico específico comparado con aquellos > 6 meses [20/51 (39.2\%) contra 5/90 (5.5\%)] con un OR de 10.9 (IC 95\%: 3.78 - 31.74). Colitis eosinofilica es más probable encontrarla en muestra de biopsias de colon derecho comparada contra colon izquierdo 9/11 (81\%) contra 6/11 (54\%) $(p=0.001)$, de igual forma colitis linfocítica 8/9 (88\%) contra 5/9 (55\%) $(p=0.001)$. Conclusiones: La utilidad de las biopsias en pacientes con DCNI y colon macroscópico normal es alta. En nuestro estudio el rendimiento fue del 17.7\% para un diagnóstico específico y estos pacientes podrían beneficiarse de un tratamiento definitivo. Estos hallazgos específicos

\section{Correspondencia:}

*Luis Alberto López-Valenzuela

Centro Médico Nacional Siglo XXI

Av. Cuauhtémoc 330

Col. Doctores, Del. Cuauhtémoc

Fecha de recepción: 09-01-2019

Fecha de aceptación: 02-03-2019

DOI: 10.24875/END.M19000009
Disponible en internet: 06-05-2019

Endoscopia. 2019;31:77-84

www.endoscopia-ameg.com

E-mail: gastro.alberto@gmail.com 0188-9893/๑ 2019. Asociación Mexicana de Endoscopia Gastrointestinal, publicado por Permanyer México SA de CV, todos los derechos reservados. 
se observaron con mayor frecuencia en pacientes con diarrea crónica no inflamatoria con una evolución < 6 meses, así como en biopsias obtenidas en colon derecho.

Palabras clave: Diarrea crónica no inflamatoria. Colonoscopia. Mucosa normal. Hallazgos histológicos.

\section{Usefulness of endoscopic biopsies in patients with chronic non-inflammatory diarrhea and normal colonoscopy}

\section{Abstract}

Introduction: Colonoscopy is part of the diagnostic arsenal in patients with chronic non-inflammatory diarrhea; however the colon mucosa may be normal. The diagnostic yield of colonoscopy is 7-31\%, but it can provide a definitive diagnosis. Aim: To evaluate the usefulness of endoscopic biopsies in patients with chronic non-inflammatory diarrhea and normal colonoscopy. Material and Methods: Retrolective, transversal, descriptive study in a period of 5 years. Men and women between 18-90 years with non-inflammatory chronic diarrhea> 4 weeks were included, with normal initial laboratory tests (hemoglobin, C-reactive protein, thyroid-stimulating hormone, albumin), and normal colonoscopy. Statistical Analysis: Qualitative variables are presented in percentages and frequencies. Qualitative variables Chi2 or Fisher's exact test was used. $P$ value less than 0.05 was considered significant. Results: 349 patients were reviewed, 141/349 met inclusion criteria. The endoscopic biopsies in normal colonoscopy had a utility for a specific histological diagnosis in 25/141 (17.7\%): eosinophilic colitis 11/141 (7.8\%), lymphocytic colitis $9 / 141$ (6.4\%), ulcerative colitis $3 / 141$ (2.1\%), Crohn's disease $1 / 141$ (0.7\%), melanosis coli $1 / 141$ (0.7\%). The evolution of diarrhea < 6 months is more likely than a specific diagnosis compared > 6 months 20/51 (39.2\%) vs 5/90 (5.5\%) OR 10.9 (95\% IC: 3.78-31.74). Eosinophilic colitis is more likely in the right colon compared to the left colon $9 / 11$ (81\%) vs 6/11 (54\%) $p=$ 0.001 , and lymphocytic colitis $8 / 9$ (88\%) vs 5/9 (55\%) $p=0.001$. Conclusions: The usefulness of the biopsies in patients with chronic non-inflammatory diarrhea and normal macroscopic colon was $17.7 \%$, these patients could benefit from a definitive treatment. Specific diagnoses were observed more frequently in patients with an evolution $<6$ months and right colon biopsies.

Key words: Chronic non-inflammatory diarrhea. Colonoscopy. Normal colonic mucosa. Histological findings.

\section{Antecedentes}

La diarrea se puede definir por un aumento de la frecuencia, consistencia, volumen, peso de las heces y/o urgencia para defecar. En el pasado se utilizaba un peso de las heces $\geq 200$ gramos al día como criterio; sin embargo, esto puede ser engañoso y difícil de cuantificar. Actualmente el concepto de diarrea a menudo se centra en la consistencia de las heces, las evacuaciones líquidas con una puntuación en la escala de Bristol $\geq 5$ define mejor el concepto de diarrea ${ }^{1}$.

La diarrea crónica se puede definir cuando esta tiene una duración $>4$ semanas, esto se ha asociado con una etiología generalmente de tipo no infeccioso y por lo tanto requiere un protocolo más profundo de investigación ${ }^{1}$. Es una patología frecuente de consulta para el gastroenterólogo, se estima una prevalencia de diarrea crónica del $1-5 \%$ en la población general y aumenta en aquellos individuos con inmunosupresión ${ }^{2,3}$. Tiene una etiología variada, como infecciones, enfermedad inflamatoria intestinal (EII), neoplasia, enfermedad diverticular, patología endocrinometabólica, inducida por fármacos, radioterapia y/o funcional ${ }^{4}$.

El diagnóstico de pacientes con diarrea crónica es un desafío; el abordaje de estos pacientes se debe iniciar de acuerdo con la sospecha clínica y el juicio del médico ${ }^{1-5}$. El primer paso es realizar una historia clínica detallada para planificar las siguientes investigaciones, el segundo paso es descartar etiología infecciosa, así como enfermedades sistémicas no controladas (disfunción tiroidea, diabetes mellitus descontrolada) ${ }^{6}$. De acuerdo a la Sociedad Británica de Gastroenterología, en las pruebas iniciales se deben realizar análisis de sangre como biometría hemática completa $(\mathrm{BHC})$, proteína c reactiva $(\mathrm{PCR})$, química sanguínea, electrolitos séricos, pruebas de función tiroidea con hormona estimulante de tiroides (TSH) y tiroxina (T4), análisis de las heces fecales con examen coprológico y coproparasitoscópico así como pruebas serológicas para descartar enfermedad celiaca ${ }^{1-7}$. Posteriormente se debe clasificar la diarrea de acuerdo a sus características macroscópicas y análisis bioquímicos (secretora, osmótica, inflamatoria o esteatorrea) $)^{1,7}$.

La colonoscopia es una de las herramientas más importantes en los métodos diagnósticos para enfermedades colorrectales, identifica lesiones tempranas de cáncer, hemorragia gastrointestinal y divertículos, entre otras ${ }^{9-10}$. Sin embargo, algunos pacientes con diarrea crónica tienen una colonoscopia normal y la toma de biopsias endoscópicas puede revelar 
información necesaria para establecer un diagnóstico específico y así preinscribir un tratamiento definitivo ${ }^{1-9}$. La colonoscopia con biopsias de la mucosa es valiosa tiene un rendimiento diagnóstico variable del $7-31 \%$ en los diferentes estudios realizados ${ }^{1,2,12}$.

De acuerdo con el Royal College of Pathologists los hallazgos histopatológicos de las biopsias colonoscópicas solo tienen un valor predictivo y clínico en pacientes con diarrea crónica acuosa no inflamatoria, el objetivo es descartar la colitis microscópica ${ }^{1,14,15}$. Se recomienda un máximo de 6 biopsias, tres biopsias en colon derecho (ciego hasta colon transverso distal) y 3 biopsias de colon izquierdo, las cuales se deben separar en dos frascos diferentes y otros estudios recomiendan hasta 12 biopsias de ambos lados derecho e izquierdo de colon ${ }^{1,11,14-17}$. La Sociedad Británica de Endoscopia Gastrointestinal que la toma de biopsias son un criterio de calidad de colonoscopia en pacientes con diarrea crónica ${ }^{10,14,20}$. Actualmente existen pocos estudios que evalúen la utilidad real de las biopsias endoscópicas en este grupo de pacientes de diarrea crónica no inflamatoria y colonoscopia normal ${ }^{16}$.

\section{Objetivo}

Evaluar la utilidad de las biopsias endoscópicas en pacientes con diarrea crónica no inflamatoria y colonoscopia normal.

\section{Material y métodos}

Se realizó un estudio retrospectivo, transversal, descriptivo y observacional entre mayo de 2013 y mayo de 2018, en el Hospital de Especialidades Centro Médico Nacional Siglo XXI. Los criterios de inclusión fueron: hombres y mujeres de entre 18 y 90 años con diarrea crónica no inflamatoria, la cual se definió con base en dos criterios: 1) con una duración mayor a 4 semanas con análisis iniciales de laboratorio normales (hemoglobina, proteína c reactiva, hormona estimulante de tiroides, albúmina), así como análisis coprológico negativo o normal, y resultados de colonoscopia normales. Los criterios de exclusión fueron: pacientes con $<4$ biopsias de cada segmento derecho e izquierdo de colon, hemorragia de tubo digestivo evidente, análisis coprológico con leucocitos fecales y/o sangre oculta en heces positiva $(\mathrm{SOH}+)$, coproparasitoscópicos seriados (3) con alguno positivo, coprocultivos positivos, anemia por déficit de hierro, Virus de Inmunodeficiencia Humana (VIH) / sida; inmunosupresión por fármacos, quimioterapia, neoplasia digestiva, Ell (Colitis Ulcerativa Crónica Idiopática o enfermedad de Crohn conocida), consumo crónico de fármacos antiinflamatorios no esteroideos (AINE) durante $>6$ semanas, cirugía previa de colon, colonoscopia incompleta y embarazo. Se eliminaron del análisis aquellos pacientes con información incompleta del resultado histológico y pacientes con histología normal.

Se recabaron los datos demográficos de los pacientes que incluyeron: sexo, edad, comorbilidades, datos clínicos (tipo de presentación, duración, características de la diarrea) y síntomas concomitantes. Se realizó un análisis de la colonoscopia de las características de la mucosa, preparación intestinal (puntaje de escala de Boston), número de biopsias realizadas por segmentos (colon derecho, que incluye ciego, colon ascendente y mitad proximal de colon transverso; colon izquierdo, que incluye recto, sigmoides, colon descendente y mitad distal de colon transverso). La mucosa de colon se consideró normal si presentaba las siguientes características: superficie lisa y regular, color rosado, con patrón vascular fino ramificado visible, con conservación de pliegues en todos sus segmentos. Se consideró anormal cualquier alteración, incluyendo zonas de eritema, pérdida del patrón vascular, úlceras, hemorragia, tumoraciones, malformaciones vasculares y divertículos ${ }^{13}$. El examen coprológico se consideró anormal si había presencia de leucocitos; sangre oculta positiva, coproparasitoscópico (CPS) anormal si había presencia de algún parásito y coprocultivo si había desarrollo de alguna bacteria patógena ${ }^{13-22}$.

La diarrea crónica se definió como la presencia de evacuaciones disminuidas en consistencia y frecuencia aumentada con una duración de más de cuatro. El termino no inflamatorio se definió en examen coprológico sin evidencia de leucocitos además clínicamente ausente de sangre en evacuaciones ${ }^{8-13}$.

Las lesiones histológicas fueron clasificadas de acuerdo a los criterios utilizados por Langner $C^{22,23,24}$ y Working Group of Digestive Diseases of the European Society of Pathology (ESP) and the European Microscopic Colitis Group (EMCG) por sus siglas en inglés ${ }^{23,35}$. 1) la colitis linfocítica se definió por el aumento de los linfocitos intraepiteliales (> 20 linfocitos por 100 células epiteliales); 2) la colitis colagenosa como inflamación crónica de la mucosa asociada con la capa fibrosa subepitelial de colágena $>10 \mu \mathrm{m}$ e involucrando capilares, glóbulos rojos y células inflamatorias; 3) la inflamación inespecífica cuando no se cumplen los criterios de inflamación especifica pero se superan los límites de la mucosa normal; 4) Ia hiperplasia linfoide como mucosa que contiene folículos linfoides activados con centro germinal agrandado; 5) la melanosis coli como la presencia de pigmentos de lipofuscina en macrófago de colon asociado con el 


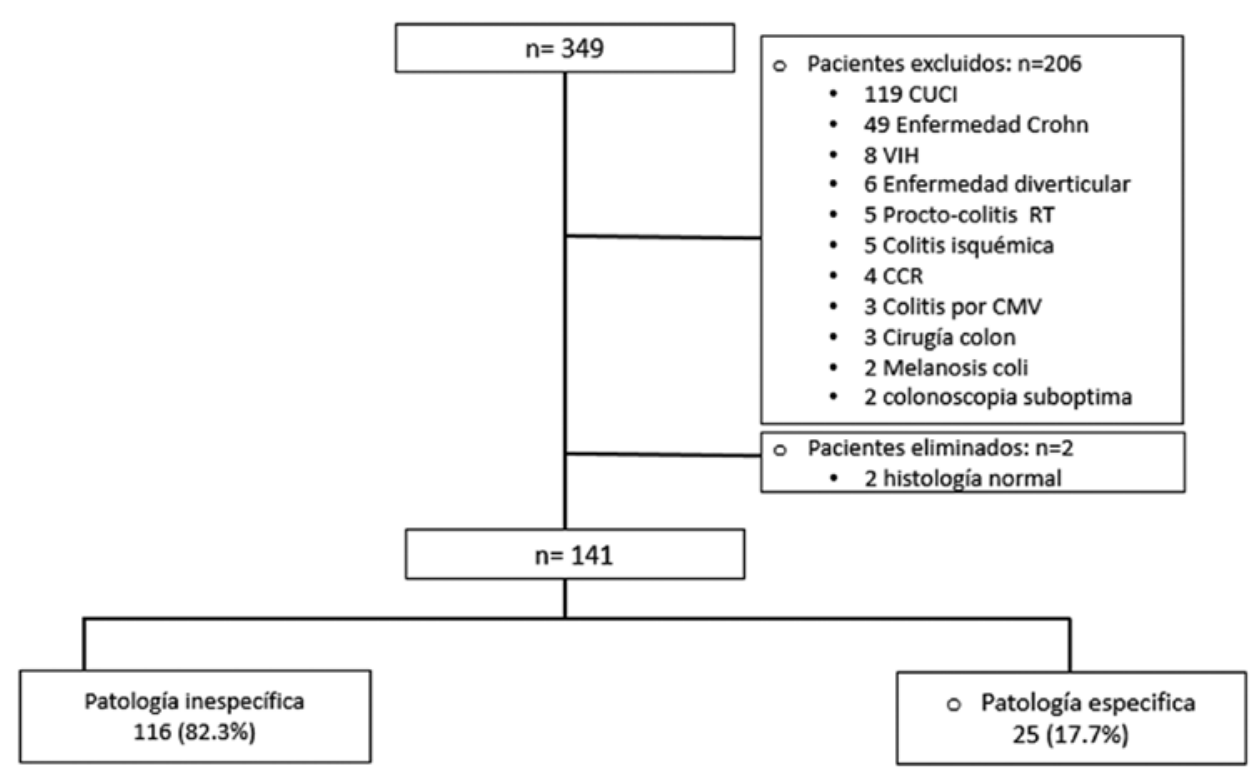

Figura 1. Pacientes con diarrea crónica.

CUCI: Colitis Ulcerativa Crónica Idiopática; VIH: Virus de Inmunodeficiencia Humana; RT: Radioterapia; CCR: Cáncer Colorrectal; CMV: Citomegalovirus.

uso de laxantes basados en antraquinona, y 6) respecto a la colitis eosinofílica, debido a que no existe una guía especifica del número de eosinófilos para definir colitis eosinofílica, utilizamos los criterios del artículo de Margaret $\mathrm{H}$. Collins (> 100 eosinófilos en colon derecho por campo de alto poder comprometiendo la submucosa y muscular propia, $>84$ en colon transverso y descendente, $>64$ en sigmoides y recto, $>35$ eosinófilos en colon se consideró anormal), la enteritis eosinofílica se definió con $>56$ eosinófilos por campo de alto poder en el epitelio del intestino delgado ${ }^{27,28}$. Las biopsias se analizaron al microscopio óptico usando hematoxilina/eosina, tricrómico de Masson para colágeno.

Con base en el estudio de Kagueyama, et al. (2014) decidimos dividir nuestra muestra de acuerdo al tiempo de evolución en dos grupos: menor y mayor a 6 meses; y comparar los hallazgos histológicos, y comparamos los hallazgos histológicos de un diagnóstico específico e inespecíficos de cada grupo.

\section{Análisis estadístico}

Las variables cuantitativas se describieron utilizando medidas de tendencia central y de dispersión de acuerdo a la distribución de los datos (media y desviación estándar para las variables paramétricas y mediana con rangos intercuartílicos 25 y $75 \%$ para variables no paramétricas). Para establecer la normalidad en la distribución se utilizó la prueba de Kolmogorov-Smirnoff. Las variables cualitativas se presentan como porcentajes y frecuencias. Para demostrar diferencias entre grupos se utilizaron las pruebas de $U$ de Mann-Whitney ot de Student para variables cuantitativas y $\mathrm{Chi}^{2}$ o prueba exacta de Fisher para cualitativas. Se utilizó una $p<0.05$ para establecer significancia estadística. Para el análisis de los datos se utilizó el paquete estadístico SPSS ${ }^{\circledR}$ versión 21.

\section{Resultados}

Se revisaron un total de 349 pacientes con diarrea crónica, se excluyeron 206 pacientes en los cuales se encontraron hallazgos clínicos, bioquímicos de diarrea inflamatoria y/o cambios macroscópicos en la colonoscopia; 119 (CUCl), y 49 enfermedad de Crohn, 8 Virus de Inmunodeficiencia Humana (VIH), 6 enfermedad diverticular, 5 proctocolitis por radioterapia, 5 colitis isquémica, 4 cáncer colorrectal, 3 citomegalovirus (CMV), 3 cirugía de colon/intestino delgado, 2 melanosis coli, 2 colonoscopias subóptimas (preparación inadecuada con escala de Boston $<5$ puntos) y 2 pacientes con histología normal fueron eliminados del estudio. Un total de 141 pacientes cumplieron con los criterios y fueron incluidos en el análisis; la colonoscopia y la toma de biopsias tuvieron una utilidad para un diagnóstico histológico específico en 25/141 (17.7\%), y patología inespecífica en 116/141 (82.3\%) (Fig. 1). 


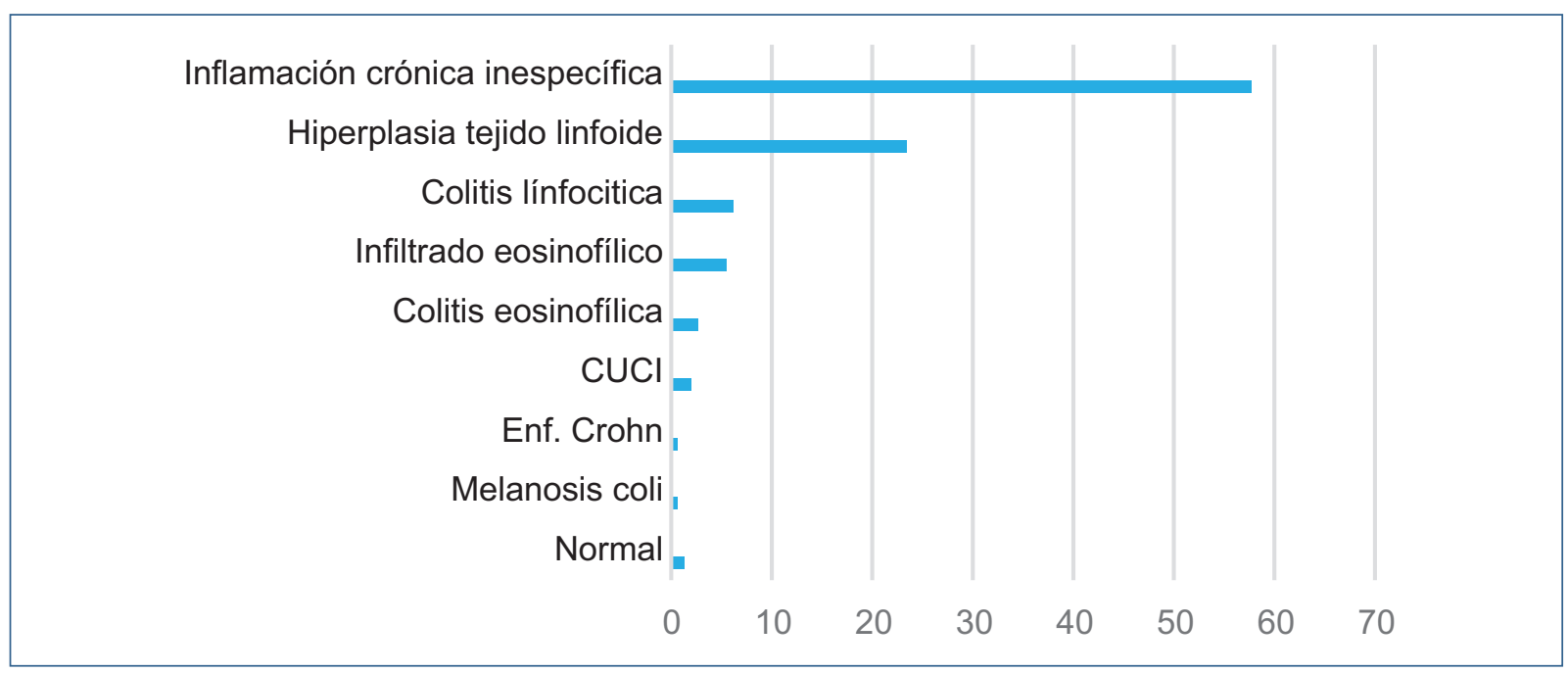

Figura 2. Hallazgos histológicos en mucosa de colon.

Solo $141 / 349$ (40.4\%) pacientes cumplieron con los criterios de inclusión, 98/141 (69.5\%) fueron mujeres y 43/141 (30.5\%) hombres; la mediana de edad fue 52 años (rango: 18-82 años), 51/141 (36.2\%) con tiempo de evolución de la diarrea $<6$ meses y 90/141 (63.8\%) con evolución $>6$ meses, mediana de hemoglobina $14 \mathrm{~g} / \mathrm{dl}( \pm 2.1)$, media de albúmina $4.2( \pm 0.4)$. Media de la limpieza de colon medida por escala de Boston, $7.4( \pm 1.1)$ (Tabla 1).

De los hallazgos histológicos en mucosa de colon, los más frecuente fueron: inflamación crónica inespecífica $83 / 141$ (58.8\%), hiperplasia de tejido linfoide $33 / 141$ (23.5\%) y patologías específicas 25/141 (17.7\%). De las patologías específicas, la más frecuente fue la colitis eosinofílica 11/141 (7.8\%), seguida de colitis linfocítica 9/141 (6.4\%), CUCI 3/141 (2.1\%), enfermedad de Crohn $1 / 141(0.7 \%)$ y melanosis coli $1 / 141(0.7 \%)$ (Fig. 2).

La intubación de íleon terminal se llevó a cabo en $138 / 141(97.9 \%)$ y se realizó biopsia de íleon terminal en 131/141 (92.9\%). Se realizó panendoscopia con toma de biopsias de duodeno a 31/141 (22\%). Los hallazgos de íleon terminal fueron: inflamación crónica inespecífica, 80/131 (61.0\%); hiperplasia del tejido linfoide, 30/131 (22.9\%); histología normal, 16/131 (12.3\%). Los hallazgos específicos en íleon terminal fueron del $5 / 131$ (3.8\%); infiltrado eosinofílico anormal 3/131 (2.3\%) e infiltrado de linfocitos anormal (> 5 linfocitos por campo de alto poder) 2/131 (1.5\%); sin embargo, el $100 \%$ de los pacientes tenía estos mismo hallazgos en colon (colitis eosinofílica y/o colitis linfocítica). En los 31/141 pacientes a quienes se realizó biopsia de duodeno no se encontraron hallazgos específicos, incluso
Tabla 1. Datos demográficos

\begin{tabular}{|l|c|}
\hline Variable & Resultados ( $\mathbf{n}=\mathbf{1 4 1})$ \\
\hline Edad & 52 años (18-82) \\
\hline Sexo & $69.5 \%$ mujeres \\
\hline Evolución $<6$ meses & $36.2 \%$ \\
\hline Evolución $>6$ meses & $63.8 \%$ \\
\hline Hemoglobina & $14 \mathrm{~g} / \mathrm{dl}( \pm 2.1)$ \\
\hline Albúmina & $4.2( \pm 0.4)$ \\
\hline Limpieza colon (Boston) & $7.4( \pm 1.1)$ \\
\hline
\end{tabular}

en los pacientes que presentaban algún hallazgo específico en colon (Tabla 2).

Los hallazgos histológicos para una patología específica de acuerdo con el tiempo de evolución de la diarrea, fue 20/51 (39.2\%) para < 6 meses frente a $5 / 90(3.4 \%)$ en aquellos con $>6$ meses, con un OR de 10.9 (IC 95\%: 3.78-31.74), para una patología inespecífica $31 / 51(60.8 \%)$ comparado con $85 / 90(96.5 \%)$, con un valor de $p=0.001$ cuando es $<6$ meses $y>6$ meses (Tabla 3).

Cuando se compararon de forma general los hallazgos histológicos del colon derecho contra los de colon izquierdo, se observó que son ligeramente más efectivas las del colon derecho, con un $14.2 \%$ frente a un $10.6 \%$ (Fig. 3). Cuando se comparó solo en los pacientes con patología específica $25 / 141$ se observó que el colon derecho fue superior al colon izquierdo 21/25 (84\%) contra 15/25 (60\%) (Tabla 4).

Los diagnósticos más comunes fueron colitis eosinofílica $11 / 141$ (7.8\%) y colitis linfocítica $9 / 141$ (6.4\%). 


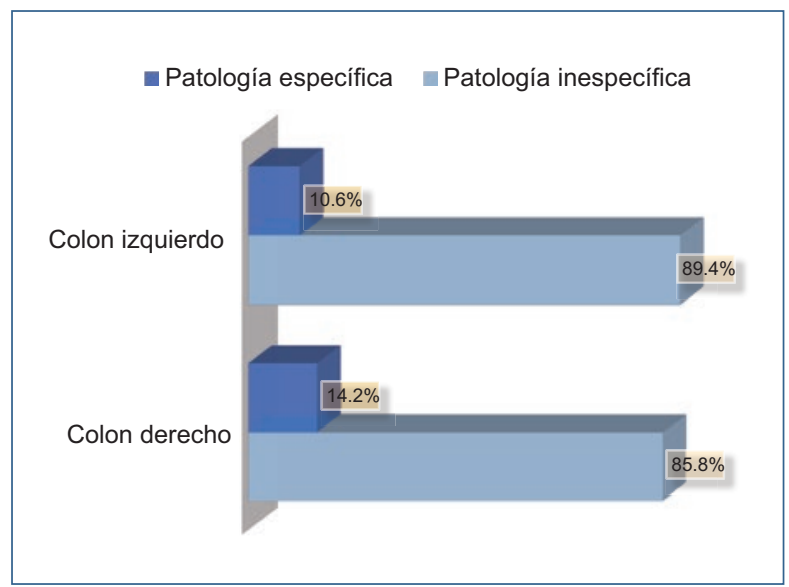

Figura 3. Comparación entre segmentos de colon y hallazgos histológicos.

Tabla 2. Biopsias de íleon, duodeno y mucosa de colon

\begin{tabular}{|l|c|}
\hline Características & Resultados \\
\hline Intubación del íleon terminal & $97.9 \%$ \\
\hline Biopsias del íleon terminal & $92.9 \%$ \\
\hline Biopsia de duodeno & $22 \%$ \\
\hline $\begin{array}{l}\text { Hallazgos histopatológicos específicos } \\
\text { en íleon terminal }\end{array}$ & $3.8 \%$ \\
\hline $\begin{array}{l}\text { Hallazgos histopatológicos específicos } \\
\text { en duodeno }\end{array}$ & $0 \%$ \\
\hline $\begin{array}{l}\text { Hallazgos histopatológicos específicos } \\
\text { en colon derecho }\end{array}$ & $14.1 \%$ \\
\hline $\begin{array}{l}\text { Hallazgos histopatológicos específicos } \\
\text { en colon izquierdo }\end{array}$ & $10.5 \%$ \\
\hline
\end{tabular}

Se relacionó los hallazgos específicos de las biopsias por segmentos de colon y se encontró que la colitis eosinofílica es más probable encontrarla en biopsias de colon derecho $9 / 11$ (81\%) comparada con $6 / 11(54 \%)$ con el colon izquierdo con un valor de $p=0.001$, de forma similar la colitis linfocítica en colon derecho 8/9 (88\%) comparada con 5/9 (55\%) en colon izquierdo con un valor de $p=0.001$. Así como enfermedad de Crohn es más frecuente en colon derecho; no así para $\mathrm{CUCl}$ que es más frecuente en el colon izquierdo (Tabla 4). La utilidad de las biopsias para colitis linfocítica fue del $6.4 \%$ y con predominio en mujeres hasta un $77 \%$.

\section{Discusión}

Existen controversias sobre tomar biopsias de rutina en pacientes con diarrea crónica y colonoscopia con
Tabla 3. Comparación de acuerdo a la evolución entre grupos $<6$ meses y $>6$ meses

\begin{tabular}{|l|c|c|c|}
\hline & $<\mathbf{6}$ meses & $>\mathbf{6}$ meses & $\mathbf{p}^{*}$ \\
\hline Patología específica & $20 / 51$ & $5 / 90$ & $<0.001^{*}$ \\
\hline Patología inespecífica & $39.2 \%$ & $3.4 \%$ & \\
& $31 / 51$ & $85 / 90$ & $<0.001^{*}$ \\
\hline Total $\mathrm{n}=141$ & $60.8 \%$ & $96.5 \%$ & \\
& 51 & 90 & \\
\hline
\end{tabular}

*Prueba $\mathrm{Y}^{2}$

Tabla 4. Comparación de segmentos derecho e izquierdo con patología específica $(n=25)$

\begin{tabular}{|l|c|c|c|}
\hline & $\begin{array}{c}\text { Colon } \\
\text { derecho }\end{array}$ & $\begin{array}{c}\text { Colon } \\
\text { izquierdo }\end{array}$ & p $^{*}$ \\
\hline $\begin{array}{l}\text { Colitis linfocítica } \\
\mathrm{n}=9\end{array}$ & $8 / 9(88 \%)$ & $5 / 9(55 \%)$ & $<0.001^{*}$ \\
\hline $\begin{array}{l}\text { Colitis eosinofílica } \\
\mathrm{n}=11\end{array}$ & $9 / 11(81 \%)$ & $6 / 11(54 \%)$ & $<0.001^{*}$ \\
\hline $\begin{array}{l}\mathrm{CUCl} \\
\mathrm{n}=3\end{array}$ & $2 / 3(66.6 \%)$ & $3 / 3(100 \%)$ & $<0.001^{*}$ \\
\hline $\begin{array}{l}\text { Enfermedad de } \\
\text { Crohn } \\
\mathrm{n}=1\end{array}$ & $1 / 1(100 \%)$ & $0 / 1(0 \%)$ & $<0.001^{*}$ \\
\hline $\begin{array}{l}\text { Melanosis coli } \\
\mathrm{n}=1\end{array}$ & $1 / 1(100 \%)$ & $1 / 1(100 \%)$ & $\mathrm{NS}^{*}$ \\
\hline $\begin{array}{l}\text { Total } \\
\mathrm{n}=25\end{array}$ & $21 / 25$ & $15 / 25$ & \\
\hline * Prueba Y2 , NS: No significativa. & $60 \%$ & \\
\hline
\end{tabular}

mucosa de colon normal. Muchos médicos prefieren tomar biopsias de todos los segmentos del colon e íleon terminal para descartar causas orgánicas ${ }^{16,17,20}$; Con frecuencia se encuentran hallazgos histopatológicos que ameritan establecer medidas terapéuticas específicas y necesidad de un seguimiento clinico ${ }^{14}$. Algunos autores informaron que las biopsias deben tomarse sistemáticamente, mientras que otros discuten la relación costo-efectividad del procedimiento ${ }^{17-30,33}$. En nuestro estudio a todos los pacientes se les realizó toma de cuatro o más biopsias por segmentos derecho e izquierdo, como recomiendan el Royal College of Pathologists y la Sociedad Británica de Endoscopia Gastrointestinal, que actualmente considera la toma de biopsias como un criterio de calidad en pacientes con diarrea crónica ${ }^{10,11,20}$. A pesar de la apariencia endoscópica normal de la mucosa de colon ascendente, transverso, colon descendente, recto-sigmoides e íleon terminal, más del $95 \%$ de los 
pacientes de nuestro estudio mostraron un hallazgos histológicos; este hallazgo es mayor a lo reportado en un estudio previo de Badary, et al. (2017), que fue del $82 \%$.

Nuestros hallazgos histológicos para una patología especifica fue del $17.7 \%$ y son menores en comparación con otros estudios, como el de Shah, et al. (2001), con un 31\% para diagnóstico específico, y el de Kagueyama, et al. (2014), con un $21 \%$; cabe mencionar que en el primero de estos estudios hasta el $27 \%$ de sus pacientes tenían colonoscopia anormal. Esto es distinto a lo reportado por Rafi, et al. (2008), quienes concluyen que las biopsias por segmento de colon en pacientes con diarrea crónica y colonoscopia normal son de poco valor diagnóstico.

Llama la atención que en 4 pacientes se emitió un reporte histopatológico compatible con EII (3 con CUCI y 1 Enfermedad de Crohn), La sola biopsia endoscópica es insuficiente para establecer el diagnóstico definitivo de algún tipo de Ell. A pesar de ello, estudios previos han publicado hallazgos histológicos que sugieren ElI ${ }^{13,14,16,20}$. Sin embargo, la Ell no tiene un estándar de oro para diagnóstico. Debe considerarse que la melanosis coli no es una patología ni causa de diarrea crónica pero sugiere el uso de laxantes estimulantes que algunas veces puede ser negado por el paciente ${ }^{16-33}$.

En relación con el estudio de Kegueyama (2014), decidimos dividir a los pacientes en dos grupos de acuerdo al tiempo de evolución en $<6$ meses y $>6$ meses, y nuestro estudio demuestra que los hallazgos histológicos específicos son más comunes cuando la evolución de la DCNI es $<6$ meses comparado con aquellos que tienen una duración $>6$ meses (39.2 frente a $3.4 \%$ ) con una OR de 10.9 (IC 95\%: 3.78-31.74), lo cual nos indica que aquellos pacientes con una evolución $<6$ meses tiene diez veces mayor probabilidad de encontrar un diagnóstico específico y establecer un tratamiento dirigido. Los hallazgos histológicos del coIon derecho son más frecuentes cuando se comparan con los de colon izquierdo en patologías específicas (84\% contra $60 \%$ ), esto difiere a lo reportado por Fine, $\mathrm{KD}$ et al. ${ }^{31}$ que comentan que la sola rectosigmoidoscopia con toma de biopsias proporciona un diagnóstico en el $99 \%$ de los casos. Nuestro estudio apoya que en este grupo de pacientes se debe realizar una colonoscopia completa con biopsias de colon derecho e izquierdo ${ }^{19-35}$. La colitis linfocítica al igual que la colitis eosinofílica tienen una mayor probabilidad de diagnóstico en las biopsias del lado derecho comparadas contra las de lado izquierdo.

En nuestro estudio el rendimiento global para colitis linfocítica fue de $6.4 \%$ y es similar a lo reportado por
Shah RJ, et al..$^{12}$ de $7 \%$, pero más bajo a lo reportado por Carmona-Sánchez R, et al. ${ }^{13}$ de $11 \%$, la colitis microscópica representa uno de los principales motivos por los cuales se debe realizar biopsia en este grupo de pacientes ${ }^{24,25,29}$. El hallazgo específico más frecuente en nuestro estudio fue colitis eosinofílica, en un $7.8 \%$, que es más bajo a lo reportado por Kagueyama, et al. ${ }^{13}$ (2014); que actualmente no hay un consenso de criterios diagnósticos para esta patología, sin embargo los hallazgos endoscópicos son cambios inflamatorios leves con edema, eritema, pérdida del patrón vascular, las biopsias generalmente revelan infiltrado eosinofílico de la lámina propia que se extiende hasta la capa muscular, cabe mencionar que la mucosa y submucosa en una persona sana tiene entre 5-35 eosinófilos por campo de alto poder y no indica enfermedad ${ }^{14,15}$.

Los hallazgos para una patología específica en íleon terminal en nuestro estudio son bajos del 3.8\%, sin embargo el $100 \%$ de los pacientes tenía estos mismo hallazgos en colon y el porcentaje es muy similar a lo reportado por Shah RJ, et al. (2001). En nuestro estudio, las biopsias de colon nos permiten identificar hallazgos histológicos específicos en diarrea crónica no inflamatoria y mucosa de colon normal en el $17.7 \%$ y estos pacientes podrían tratarse con una terapia especifica de acuerdo a los hallazgos histológicos (colitis linfocítica, colitis eosinofílica e infiltrado linfoide, $\mathrm{CUCl}$, enfermedad de Crohn, melanosis coli). La colitis linfocítica puede tratarse con esteroides, la mejor respuesta se obtiene con budesonida, pero la recurrencia es muy frecuente al suspender el tratamiento.

\section{Conclusiones}

La utilidad de las biopsias en pacientes con diarrea crónica no inflamatoria y colon macroscópico normal es alta, lo cual justifica la toma de biopsias protocolizadas de colon derecho e izquierdo. En nuestro estudio el rendimiento fue del $17.7 \%$ para un diagnóstico definitivo y estos pacientes podrían beneficiarse de un tratamiento específico de acuerdo a los hallazgos histológicos. Los diagnósticos específicos se observaron con mayor frecuencia en pacientes con diarrea crónica de evolución menor de 6 meses comparados con aquellos que tienen una evolución mayor de 6 meses. También se observaron estos hallazgos histológicos específicos más frecuentemente en colon derecho comparados con colon izquierdo. La patología más frecuente en nuestro estudio fue la colitis eosinofílica, seguido de colitis linfocítica. 


\section{Bibliografía}

1. Schiller L, Pardi D, Spiller R, Semrad CE, Surawicz CM, Giannella RA et al. Gastro 2013 APDW/WCOG Shanghai working party report: chronic diarrhea: definition, classification, diagnosis. J Gastroenterol Hepatol. 2014;29:6-25.

2. Schiller L. Definitions, pathophysiology, and evaluation of chronic diarrhoea. Best Pract Res Clin Gastroenterol. 2012;26(5):551-62.

3. Schiller L. Evaluation of chronic diarrhea and irritable bowel syndrome with diarrhea in adults in the era of precision medicine. Am J Gastroenterol. 2018;113(5):660-9.

4. Camilleri M, Sellin J, Barrett E. Pathophysiology, evaluation, and management of chronic watery diarrhea. Gastroenterology. 2017;152:515-32.

5. Dosanjh G, Pardi DS. Chronic unexplained diarrhea: a logical and cost-effective approach to assessment. Curr Opin Gastroenterol. 2016;32(1):55-60.

6. Steffer KJ, Santa Ana CA, Cole JA, Fordtran JS. The Practical Value of Comprehensive Stool Analysis in Detecting the Cause of Idiopathic Chronic Diarrhea. Gastroenterol Clin North Am. 2012;41(3):539-60.

7. Shen B, Khan K, Ikenberry SO, Anderson MA, Banerjee S, Baron T, et al. The role of endoscopy in the management of patients with diarrhea. Gastrointest Endosc. 2010;71(6):887-92.

8. Thomas PD, Forbes A, Green J, Howdle P, Long R, Playford R, et al Guidelines for the investigation of chronic diarrhoea, 2nd edition. Gut 2003;52(5):1-15.

9. Schiller LR. Chronic diarrhea: to biopsy or not to biopsy. Gastrointest Endosc. 2005;61(3):376-7.

10. Shah R, Preiser C, Bleau B, et al. Usefulness of colonoscopy with biopsy in the evaluation of patients with chronic diarrhea. Am Coll Gastroenterology. 2001:96(4):1091-5.

11. Carmona-Sánchez R, Tostado-Fernández F, Esmer-Sánchez D. La utilidad de la colonoscopia con toma de biopsias en el estudio de pacientes con diarrea crónica. Rev Gastroenterol Mex. 2007;72(4):349-54.

12. Badary D, Hafez M. Role of mucosal colonic biopsy in patients with chronic unexplained diarrhoea who their colonoscopy is normal. 2017;6:185-89.

13. Kagueyama $F$, Nicoli $F$, Bonatto $M$, et al. Importance of biopsies and histological evaluation in patients with chronic diarrhea and normal colonoscopies. Arq Bras Cir Dig. 2014;27:184-7.

14. Da Silva JG, De Brito T, Cintra DA, Laudanna AA, Sipahi AM. Histologic study of colonic mucosa in patients with chronic diarrhea and normal colonoscopic findings. J Clin Gastroenterol. 2006;40(1):44-8.

15. Marshall J, Singh R, Diaz A. Chronic, unexplained diarrhea: are biopsies necessary if colonoscopy is normal? Am J Gastroenterol. 1995:90:372-6.

16. Makkar R, Lopez R, Shen B. Clinical utility of retrograde terminal ileum intubation in the evaluation of chronic non-bloody diarrhea. J Dig Dis. 2013;14(10):536-42.

17. Harewood G, Olson J, Mattek N, et al. Colonic biopsy practice for evaluation of diarrhea in patients with normal endoscopic findings: results from a national endoscopic database. Gastrointest Endosc. 2005; 61(3):371-5.

18. Elliot V, Bateman A, Green B. The endoscopically normal colon when is mapping biopsy histopathologically justifiable? Frontline Gastroenterol. 2012;3:104-8.

19. Greenson JK. Inflammatory/Descriptive/latrogenic Colitides. En: John R. Goldblum, editor. Gastrointestinal and Liver Pathology. Second edition. Elsevier; 2012. p.367-89.

20. Langner $\mathrm{C}$. Colorectal normal histology and histopathologic findings in patients with chronic diarrhea. Gastroenterol Clin North Am. 2012;41: 561-80.

21. Langner C, Aust D, Ensari A, Villanacci V, Becheanu G, Miehlke S, et al. Histology of microscopic colitis review with a practical approach for pathologists. Histopathology. 2015;66(5):613-26.

22. Gentile N, Yen EF. Prevalence, pathogenesis, diagnosis, and management of microscopic colitis. Gut and Liver. 2018;12(3):227-35.

23. Pardi DS. Diagnosis and management of microscopic colitis. Am J Gastroenterol. 2017;112(1):78-85

24. Nguyen GC, Smalley WE, Vege SS, Carrasco-Labra A, Flamm SL, Gerson L, et al. American Gastroenterological Association Institute Guideline on the Medical Management of Microscopic Colitis. Gastroenterology. 2016;150(1):242-6.

25. Collins MH. Histopathologic features of eosinophilic esophagitis and eosinophilic gastrointestinal diseases. Gastroenterol Clin North Am. 2014;43(2):257-68

26. Alfadda AA, Storr MA, Shaffer EA. Eosinophilic colitis: epidemiology, clinical features, and current management. Ther Adv Gastroenterol. 2010;4(5):301-9

27. Tack J. Functional Diarrhea. Gastroenterol Clin North Am. 2012;41(3): 629-37.

28. Santos A, Reis DM. Microscopic analysis of patients with chronic diarrhea without macroscopic disease. J Coloproctol (Rio J). 2016;36(1):21-6.

29. Fine KD, Seidel RH, Do K. The prevalence, anatomic distribution, and diagnosis of colonic causes of chronic diarrhea. Gastrointest Endosc. 2000;51(3):318-26.

30. Prior A, Lessells AM, Whorwell PJ. Is biopsy necessary if colonoscopy is normal? Dig Dis Sci. 1987;32(7):673-6.

31. Ramadan A, Fattah WA, El-Kashiski KA. The Yield of Mapping Biopsy from Apparent Endoscopically Normal Colon and Correlation with Symptoms. Journal of Gastroenterology and Hepatology Research. 2014; 3(7):1168-72.

32. Abdullah M, Firmansyah MA. Clinical Approach and Management of Chronic Diarrhea. Acta Med Indones. 2013;45(2):157-65.

33. Ud R, Zakaria M, Abid MU. Chronic Diarrhea: Large Gut Causes. Professional Med J Dec. 2008;15(4):479-85.

34. Schiller LR. Chronic diarrhea. Gastroenterology. 2004;127(1):287-93.

35. Patel Y, Pettigrew NM, Grahame GR, Bernstein CN. The diagnostic yield of lower endoscopy plus biopsy in nonbloody diarrhea. Gastrointest Endosc. 1997;46(4):338-43 